\title{
A quantitative (stereological) study of the effects of experimental unilateral cryptorchidism and subsequent orchiopexy on spermatogenesis in adult rabbit testis
}

\author{
R. D. Zhang ${ }^{1}$, X. H. Wen ${ }^{1}$, L. S. Kong ${ }^{1}$, X. Z. Deng'르, B. Peng ${ }^{1}$, \\ A. P. Huang ${ }^{1}$, Y. Wan ${ }^{3}$ and Z. W. Yang ${ }^{1 *}$ \\ ${ }^{1}$ Morphometric Research Laboratory, North Sichuan Medical College, Sichuan, China; \\ ${ }^{2}$ Department of Urological Surgery and ${ }^{3}$ Operation Unit, Affiliated Hospital, North Sichuan \\ Medical College, Sichuan, China
}

The aim of this study was to examine the controversial effects of experimental unilateral cryptorchidism and subsequent orchiopexy on the number of germ cells and other morphometric characteristics of testicular and epididymal structures in adult rabbits. Unilateral cryptorchidism was induced in $\mathbf{1 1}$ mature male New Zealand white rabbits by returning one testis, together with the ipsilateral epididymis, to the abdominal cavity via a surgical procedure. After 3 months, testes and epididymides were removed from six animals (and from six age-matched control animals that did not undergo the surgery). Orchiopexy was performed on the five remaining animals and the testes and epididymides of these animals (and an additional six agematched control animals) were removed 7 weeks later. A contemporary, unbiased and efficient stereological tool, the optical disector, was used to estimate the number of

\section{Introduction}

Despite general agreement that cryptorchidism leads to spermatogenic damage and that orchiopexy improves spermatogenesis, the extent of the damage or improvement remains obscure. How many germ cells, and in particular spermatogenic stem cells (that is, spermatogonia) survive the cryptorchid state? To what extent is spermatogenesis recovered with subsequent orchiopexy? Does unilateral cryptorchidism in one testis substantially affect spermatogenesis in the contralateral scrotal testis? The resolution of these questions is likely to depend on two major factors: (i) the cryptorchid models and (ii) the techniques used to evaluate spermatogenesis.

In humans, ectopic testes are most commonly extraabdominal, whereas, in experimentally or naturally cryptorchid animals, the ectopic testis is usually intraabdominal. Spermatogenic damage appears to be related to the position of the testis (Saito and Kumamoto, 1989; Rune

*Correspondence

Email: zwyang@nc-public.sc.cninfo.net nuclei in the testis and epididymis using methacrylateembedded sections of $25 \mu \mathrm{m}$ in thickness. Cryptorchidism resulted in severe testicular atrophy and spermatogenic arrest: type A spermatogonia and Sertoli cells only were seen in the seminiferous epithelium, and the number of type A spermatogonia per testis was reduced by $84 \%$. After orchiopexy, the testis remained atrophied and the number of type A spermatogonia returned to the nearnormal range in four of five animals, but spermatogenesis was recovered only partially at the stage of early primary spermatocytes (one animal), late primary spermatocytes (two animals) or spermatids (one animal). In conclusion, cryptorchidism caused severe spermatogenic arrest that was potentially recoverable (in view of the restoration of the number of type A spermatogonia), but orchiopexy failed to induce full recovery of spermatogenesis. et al., 1992; Rogers et al., 1998); therefore, comparison of data from different sources will result in controversy. Moreover, in studies on naturally cryptorchid animals or patients, it is difficult to conclude whether any spermatogenic lesions associated with cryptorchidism are due to inherent defects in germ cells or whether they are the effects of the abnormal location of the testis. This uncertainty also creates doubts about the effect of orchiopexy, the surgical relocation of the undescended testis into the scrotum, on spermatogenic restoration, and the effects of a cryptorchid testis on the contralateral testis.

Experimental cryptorchidism in immature animals has been used to provide an analogous situation to the clinical situation in humans. As the deleterious effect of the abnormal location of the testis on germ cells or on spermatogenesis may be more severe after initiation of spermatogenesis (Penson et al., 1997) or on advanced germ cells such as spermatocytes and spermatids (Yin et al., 1997), as is observed with radiation or chemotherapy, to which differentiating cells are most sensitive, cryptorchid models of mature animals are useful for evaluation of the effects of relocation of the testis on spermatogenesis. In 
addition, such adult models are essential to verify the assumption that artificial cryptorchidism is a potential method of male contraception (retractile and intermittent cryptorchidism) (Mieusset et al., 1985) or spermatogenic protection from chemotherapeutic damage (Boekelheide et al., 1990).

Spermatogenesis in the cryptorchid testis has usually been described either in a qualitative (subjective) manner or by using semi-quantitative data, such as nuclear profile (two-dimensional) number of one type or all types of germ cell per tubule section or cross-section (Rune et al., 1992; Cortes et al., 1996; Gracia et al., 1998; Huff et al., 2001), the percentage of tubule sections containing spermatogonia (Bar-Maor et al., 1979) or elongated spermatids (Lunstra and Schanbacher, 1988), tubular fertility scores based on the presence or absence of different types of germ cell (Salman et al., 1988; Pryor et al., 1989), or the percentage of haploid cells in the testis, as measured by flow cytometry (Quinn, 1991) or fine-needle aspiration cytology (Foresta et al., 1996). These figures do not represent the real number of cells (nuclei) per organ and are not comparable between studies or statistically unbiased (Wreford, 1995), thus the statistical significance detected is questionable, especially when the absolute difference between data is small and the individual variation is large. However, an unbiased and efficient stereological tool for estimating the number of nuclei, the optical disector, has recently become available and has been used in the quantitative study of spermatogenesis in monkeys (Zhengwei et al., 1997, 1998a; Wen and Yang, 2000), men (Zhengwei et al., 1998b) and rats (Wen and Yang, 2000; Wen et al., 2000).

The aim of the present study was to examine the controversial effects of experimental unilateral cryptorchidism and subsequent orchiopexy on the testis of adult rabbits. Unilateral cryptorchidism was induced surgically for 13 weeks in mature rabbits, followed by orchiopexy for 7 weeks. The absolute (total) numbers of all types of germ cell and of Sertoli cells per testis were estimated using the optical disector approach; other quantitative parameters, such as tubule diameter and tubule length per testis, were also obtained using other stereological methods. The histology of the epididymis was also observed to confirm the spermatogenic status in the testis, and immunocytochemistry against proliferating cell nuclear antigen (PCNA) was performed to determine the proliferating activity of spermatogonia that survived after the surgery.

\section{Materials and Methods}

\section{Animals and experimental design}

Twenty-six mature male New Zealand white rabbits, aged 4-5 months and weighing an average of $2.5 \mathrm{~kg}$, were obtained from the Animal Center sponsored by the Sichuan Administrative Committee of Experimental Animals.

Cryptorchidism group. Twelve animals (body weight $2.4-3.3 \mathrm{~kg}$ ) were obtained in June and divided randomly into different subgroups. Unilateral cryptorchidism was induced in six animals by returning the left or right (chosen alternatively) testis and the ipsilateral epididymis to the abdominal cavity in a surgical procedure. The other six animals served as age-matched controls and did not undergo surgery. Thirteen weeks (92 days) later, all testes and epididymides were removed from the cryptorchid animals, and the left or right (chosen alternatively) testis and the ipsilateral epididymis were removed from the control animals.

Orchiopexy group. Fourteen animals (body weight $2.1-2.8 \mathrm{~kg}$ ) were obtained in December and divided randomly into different subgroups. Two animals were killed immediately, and testicular and epididymal sections were taken to check the spermatogenic status at this age. (The results showed that all types of germ cell were present in the testis and that spermatozoa were present in the epididymis.) Unilateral cryptorchidism was induced in six animals, as described above, and the other six animals served as agematched controls and did not undergo surgery. Thirteen weeks (91 days) later, the abdominal testis and epididymis were removed from one cryptorchid animal to check the histology of the testis and epididymis at this stage. The remaining five cryptorchid animals underwent orchiopexy. Seven weeks (49 days) later organs were removed, as described above.

\section{Surgery}

All operations were performed by an experienced surgeon and anaesthesia was induced by intra-abdominal injection of ketamine hydrochloride (in a $1 \mathrm{ml}$ bottle containing $50 \mathrm{mg}$ ketamine hydrochloride and diluted with $4 \mathrm{ml}$ saline before use; $6-7 \mathrm{mg} \mathrm{kg}^{-1}$ body weight).

Artificial unilateral cryptorchidism. One testis together with the ipsilateral epididymis and spermatic cord were placed in the abdominal cavity through an inguinal incision and the inguinal ring was closed with a suture. The contralateral organs remained in the scrotum; an inguinal hypodermic cut was made on this side to serve as a sham operation.

Orchiopexy. The abdominal testis and epididymis were returned to the scrotum through an inguinal incision; sutures were used to close the inguinal ring and to connect the organs to the scrotum.

Organ removal. Organs were removed through an abdominal incision. The testis and epididymis from the animals that underwent orchiopexy were surrounded by some adhesions, which were removed carefully after fixation. Other scrotal and abdominal organs had no adhesions or necrotic patches on their surfaces.

\section{Tissue processing}

After removal, the testis and epididymis were immersionfixed in Bouin's fluid for $24 \mathrm{~h}$ and immersed in $70 \%$ ethanol 
for 2 days. The intact organs were weighed using an electronic balance (HR-120; A\&D Company Ltd; accuracy $0.1 \mathrm{mg}$ ) to calculate the volume of the organs by dividing the weight by the density, which was determined by immersion in graded ethanol solutions of known density (Hodgman et al., 1958) and was consistently found to be $0.93 \mathrm{~g} \mathrm{ml}^{-1}$.

Testis and epididymis were cut into parallel slices of 1-2 mm thickness. Three slices were sampled in a systematic random manner (evenly spaced) and cut into smaller blocks. Three blocks (per organ) for resin embedding from all animals and three blocks for paraffin wax embedding from animals that underwent surgery were sampled systematically for further processing. After dehydration in ethanol and butanol, blocks were embedded in methacrylate (hydroxyethylmethacrylate, HEMA) resin (Heraeus Kulzer $\mathrm{GmbH}$, Werheim/Ts) for stereology, or in paraffin wax (JUNG-Histowax; Cambridge Instruments $\mathrm{GmbH}$, Nussloch) for immunocytochemistry. One methacrylate section of $25 \mu \mathrm{m}$ thickness or paraffin wax section of $5 \mu \mathrm{m}$ thickness was cut from each block using a tungsten steel knife on a semi-automatic microtome (RM2145; Leica Instruments $\mathrm{GmbH}$, Nussloch). The average area of sections, as estimated by the simple conventional stereological point counting method, was about $38 \mathrm{~mm}^{2}$ for testis and $26 \mathrm{~mm}^{2}$ for epididymis. Methacrylate-embedded sections were stained with periodic acid-Schiff's reagent and haematoxylin.

\section{Identification and grouping of spermatogenic cells and Sertoli cells}

The spermatogenic cells and Sertoli cells in the seminiferous epithelium of the rabbit testis appeared to be similar to those seen in the monkey testis and were therefore identified and grouped on the basis of the description by Zhengwei et al. (1997) (see Fig. 1a,b).

Sertoli cells. In Sertoli cells, the nuclei were readily differentiated from germ cells by their irregular shape, paler chromatin pattern, single nucleolus and the absence of a clear cytoplasmic boundary.

Type A spermatogonia. Type A spermatogonia were scattered along the basement membrane and had a nucleus with diffuse fine or relatively small granulated chromatin.

Type B spermatogonia. Type B spermatogonia were also located on the basement membrane and their nuclei were identified and differentiated from type A spermatogonia by the presence of a centrally located nucleolus and clumps or relatively large granules of darkly stained chromatin distributed along the nuclear membrane and around the nucleolus. In addition, groups of type B spermatogonia were more often distributed in a line (Fig. 1a).
In a study on Dutch-belted rabbits, Swierstra et al. (1963) classified another category of spermatogonia in $4 \mu \mathrm{m}$ thick sections of testis fixed in Allen's fixative and probably embedded in paraffin wax. These cells were referred to as intermediate type spermatogonia. On the basis of their description of intermediate type spermatogonia, which had ovoid nuclei with the long axis parallel to the basement membrane and chromatin in the form of coarse granules, it would have been possible to include many of this type of spermatogonia in the group of type A spermatogonia, as defined in the present study.

Preleptotene, leptotene and zygotene primary spermatocytes. The nuclei of preleptotene, leptotene and zygotene primary spermatocytes were characterized by thread-like clumps of chromatin and their association with both pachytene primary spermatocytes and elongating spermatids in the normal testis, that is, preleptotene, leptotene and zygotene primary spermatocytes were usually present in association with pachytene primary spermatocytes and elongating spermatids (Fig. 1b). These cells were considered as a single group in the present study. Some early preleptotene cells had a similar appearance to type B spermatogonia and were carefully differentiated according to the slightly smaller size of the nucleus.

Pachytene primary spermatocytes. Pachytene primary spermatocytes were present throughout all stages of the seminiferous cycle. Their nuclei were larger and positioned closer to the tubule lumen than were those in preleptotene, leptotene and zygotene primary spermatocytes, and their chromatin had a mottled appearance. The late stages of primary spermatocytes (for example the diakinesis of primary spermatocytes) associated with the early stages of primary spermatocytes were also regarded as pachytene spermatocytes to ensure that the pachytene spermatocytes counted in this study were in just one seminiferous cycle in the normal testis (that is, the duration of the cells was of only one cycle).

Secondary spermatocytes. In secondary spermatocytes, the nuclei were frequently associated with late pachytene spermatocytes that had yet to divide into secondary spermatocytes or with early round spermatids that had arisen from secondary spermatocytes. The nuclei were smaller and had a finer chromatin pattern compared with those of pachytene spermatocytes. The meiotic forms of these cells were common and were also included in this group.

Spermatids. The nuclei of spermatids were readily identified on the basis of their position (close to the tubule lumen), size (much smaller than those of spermatocytes), lack of heterochromatin, shape (round to elongated and flattened) and colour (elongating or elongated spermatids 

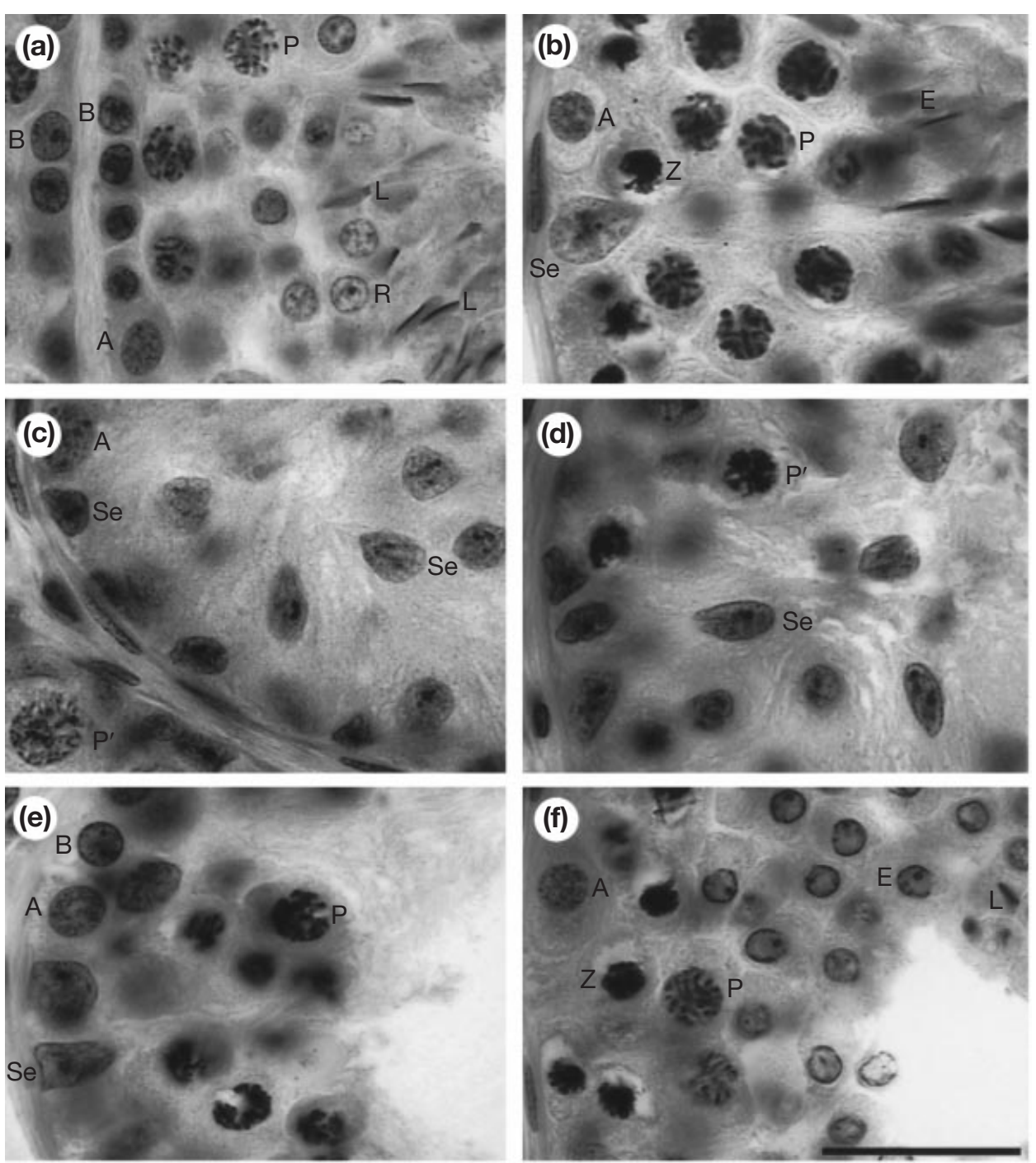

Fig. 1. Typical testicular micrographs of $25 \mu \mathrm{m}$ thick methacrylate-embedded sections of rabbit testis. (a) Section from the scrotal testis of an animal with unilateral cryptorchidism (13 weeks). (b) Section from a control animal in the orchiopexy group that did not undergo surgery. (c) Section from a cryptorchid (abdominal) testis. $(\mathrm{d}-\mathrm{f})$ Sections from the testis subjected to surgery in three animals that underwent unilateral cryptorchidism and subsequent orchiopexy (7 weeks). A: type A spermatogonia; B: type B spermatogonia; E: elongating spermatid; L: late elongated spermatid; $P$ : pachytene primary spermatocyte; $\mathrm{P}^{\prime}$ : atypical primary spermatocyte; $\mathrm{R}$ : early round spermatid; Se: Sertoli cell; Z: zygotene primary spermatocyte (nucleus). Scale bar represents $30 \mu \mathrm{m}$.

had a darker nucleus). Two major groupings were defined in this study: (i) early spermatids, consisting of early round spermatids with spherical nuclei and elongating spermatids with elongating (spherical to long and flattened) and condensing nuclei; and (ii) late elongated spermatids, which were normally associated with early round spermatids (Fig. 1a). Thus the early spermatids had a duration of just one seminiferous cycle in the normal testis.

\section{Stereology}

Number of spermatogenic cells and Sertoli cells. Sections were observed using a $\times 100$ oil immersion lens (NA1.30, UplanFl; Olympus) on an Olympus BX50 microscope. The image was captured with a video camera (WV-CP410/6; Panasonic) to a 586 computer using a video adapter (U-PMTVC; Olympus). Fields were sampled 
systematically from a random start with a computer-assisted motorized stage (Sichuan University in Chengdu and Smart Image Technology in Beijing in co-operation with Z. W. Yang) and the distance between fields was $375 \mu \mathrm{m}$. A test grid with a set of four rectangular frames (each with an area of $17 \mu \mathrm{m} \times 22 \mu \mathrm{m}$ ) was generated by a software package (Smart Image Technology in co-operation with Z. W. Yang) and superimposed on the tissue image (final magnification $\times 2677$ ). Only one frame on each field was used for counting spermatids, primary spermatocytes and Sertoli cells, whereas all four frames were used for counting spermatogonia and secondary spermatocytes.

The number of nuclei was estimated using the optical disector method as described in earlier studies (Zhengwei et al., 1997, 1998a,b; Wen and Yang, 2000; Wen et al., 2000); the number of nuclei was assumed to equate to the number of cells. In brief, $25 \mu \mathrm{m}$ methacrylate-embedded sections were sectioned optically using the $\times 100$ oil immersion lens. The upper surface of the section was brought into focus and the upper $3 \mu \mathrm{m}$ was traversed to avoid possible surface imperfections. The next $10 \mu \mathrm{m}$ of the section was examined by focusing down step by step (distance between adjacent optical sections was adjusted to be $1 \mu \mathrm{m}$ ) with the computer-assisted motorized stage, and nuclei were counted as they came into focus in the counting frame according to the disector principle (Sterio, 1984), as shown by Wreford (1995) and Wen and Yang (2000). The numerical density of each type of cell was calculated by dividing the total number of cells counted by the total volume of the disectors (volume of each disector $17 \mu \mathrm{m} \times 22 \mu \mathrm{m} \times 10 \mu \mathrm{m})$ used for the counting. The absolute number of nuclei per testis was calculated by multiplying the numerical density by the testicular volume. On average, the total number of disectors used for counting spermatogonia and secondary spermatocytes and the total number of cells counted per testis were 1080 and 933 for the scrotal testes $(n=23)$ and 1708 and 295 for the cryptorchid testes $(n=11)$ of animals that underwent surgery and control animals, respectively.

Volume of seminiferous tubules and epididymal tubules. The volume fraction (percentage) of the seminiferous tubules in the testis or the epididymal tubules in the epididymis was estimated by the conventional point counting method as described by Wen et al. (2000) and Wen and Yang (2000). The total tubule volume per organ was calculated by multiplying the fraction by the volume of the organ.

Diameter and length of seminiferous tubules. Sections were observed under a $\times 4$ objective lens (NA 0.13, UplanFl; Olympus) on the video screen at a final magnification of $\times 107$. Round or elliptical tubule profiles with a clear lumen or a clear central cytoplasmic area for atrophied tubules were sampled using a test frame according to the unbiased forbidden line rule (Gundersen,
1977) and their diameter (diameter of a circular profile or length of the shortest axis of an elliptical profile) was measured. The mean area of the tubule cross-section was calculated by multiplying the mean of squared diameters by a constant $(\pi / 4)$. The tubule length per testis was calculated by dividing the total volume of the tubules per testis by the cross-sectional area of the tubules as described by Wen et al. (2000). A total of 60 tubules was sampled and measured per testis.

\section{Immunocytochemistry}

The primary monoclonal antibody against PCNA was a product of CALBIOCHEM (Cambridge), purchased from Wuhan Boster Biological Technology Co., Ltd (China) together with a streptavidin-biotin complex detection kit. The sections were stained according to the instructions provided and diaminobenzidine (DAB) was used to develop the immunoreaction product. Omission of the primary antibody in the procedures was used as a negative control.

The number (per cross-section of the seminiferous tubules) of PCNA-positive nuclear profiles distributed along the basement membrane was determined to obtain an objective impression of the number of PCNA-positive spermatogonia that survived after the surgery. Approximately round tubule profiles (ratio between the lengths of the shortest axis and the longest axis $>0.7$ ) with a clear lumen, or a clear central cytoplasmic area for atrophied tubules, were sampled randomly as described above using a $\times 10$ objective lens, and all the PCNA-positive nuclear profiles distributed along the tubule boundary were counted at a higher magnification using a $\times 20$ objective lens. Owing to a consistent and marked difference in the number of positive cells between the testes of the animals that underwent surgery, only three tubule cross-sections were sampled per section.

\section{Statistical analyses}

Data are presented as mean \pm SEM. Comparison between the testes of individual animals that underwent surgery was made using the paired $t$ test; the unpaired $t$ test was used to detect differences between the treated animals and the agematched control animals in the same cryptorchidism group or orchiopexy group, or between the cryptorchidism group and the orchiopexy group. Significance of difference was set at $P<0.05$.

It would be inappropriate to use one-way or one-way repeated measures ANOVA to compare the three sets of data in the same treatment group (Table 1), because two of the three sets of data were obtained from the bilateral organs of the same animals and were of a statistical pairdesign, whereas the third set of data was obtained from another group of age-matched control animals. However, if one-way ANOVA was used (in conjunction with the Student-Newman-Keuls method for multiple comparisons when a significant difference was detected among the data 
Table 1. Quantitative data (mean \pm SEM) from the main testicular and epididymal structures in rabbits in the cryptorchidism and cryptorchidism plus orchiopexy groups

\begin{tabular}{|c|c|c|c|c|c|c|}
\hline \multirow[b]{2}{*}{ Parameter } & \multicolumn{3}{|c|}{ Cryptorchidism for 13 weeks } & \multicolumn{3}{|c|}{ Orchiopexy for 7 weeks } \\
\hline & $\begin{array}{l}\text { Treated } \\
(n=6)\end{array}$ & $\begin{array}{l}\text { Contra } \\
(n=6)\end{array}$ & $\begin{array}{c}\text { Control } \\
(n=6)\end{array}$ & $\begin{array}{l}\text { Treated } \\
(n=5)\end{array}$ & $\begin{array}{l}\text { Contra } \\
(n=5)\end{array}$ & $\begin{array}{c}\text { Control } \\
(n=6)\end{array}$ \\
\hline \multicolumn{7}{|l|}{ Volume $\left(\mathrm{cm}^{3}\right)$} \\
\hline Testis & $0.65 \pm 0.05^{\mathrm{a}}$ & $3.08 \pm 0.28$ & $3.04 \pm 0.16^{\mathrm{e}}$ & $0.99 \pm 0.22^{\mathrm{a}}$ & $3.09 \pm 0.23^{b}$ & $3.96 \pm 0.29$ \\
\hline Epididymis & $0.76 \pm 0.08^{a}$ & $1.31 \pm 0.11$ & $1.12 \pm 0.11$ & $0.75 \pm 0.05^{a}$ & $1.21 \pm 0.07$ & $1.45 \pm 0.12$ \\
\hline \multicolumn{7}{|l|}{ Volume per organ $\left(\mathrm{cm}^{3}\right)$} \\
\hline Seminiferous tubules & $0.26 \pm 0.05^{\mathrm{a}}$ & $2.38 \pm 0.23$ & $2.23 \pm 0.09 \mathrm{e}$ & $0.54 \pm 0.14^{\mathrm{a}}$ & $2.15 \pm 0.23^{b}$ & $3.05 \pm 0.27$ \\
\hline Epididymal tubules & $0.220 \pm 0.023^{\mathrm{a}}$ & $0.665 \pm 0.118$ & $0.581 \pm 0.056$ & $0.288 \pm 0.050^{a}$ & $0.569 \pm 0.034$ & $0.684 \pm 0.074$ \\
\hline \multicolumn{7}{|c|}{ Number of cells ( $10^{6}$ per testis) } \\
\hline A & $6.8 \pm 0.9^{\mathrm{a}}$ & $45.6 \pm 5.4$ & $37.2 \pm 5.8$ & $18.8 \pm 5.2$ & $32.3 \pm 3.2$ & $37.0 \pm 6.2$ \\
\hline B & $0.07 \pm 0.04^{\mathrm{ac}}$ & $83.49 \pm 4.81$ & $90.03 \pm 18.29$ & $15.27 \pm 7.74^{\mathrm{a}}$ & $56.37 \pm 13.15^{b}$ & $90.12 \pm 7.00$ \\
\hline $\mathrm{Pl}-\mathrm{Z}$ & o & $192 \pm 42$ & $121 \pm 34$ & $48 \pm 21^{\mathrm{a}}$ & $149 \pm 26$ & $149 \pm 26$ \\
\hline $\mathrm{P}$ & o & $399 \pm 40^{d}$ & $338 \pm 23$ & $50 \pm 20^{a}$ & $265 \pm 24^{b}$ & $409 \pm 27$ \\
\hline $\mathrm{R}-\mathrm{E}$ & o & $1161 \pm 147$ & $902 \pm 55^{\mathrm{e}}$ & $22 \pm 22^{a}$ & $931 \pm 141^{b}$ & $1401 \pm 115$ \\
\hline $\mathrm{L}$ & o & $525 \pm 60^{b}$ & $322 \pm 44$ & $\mathrm{o}$ & $424 \pm 79$ & $489 \pm 43$ \\
\hline Sertoli cells & $70.5 \pm 10.6$ & $92.4 \pm 12.1$ & $75.0 \pm 7.8$ & $74.0 \pm 19.9$ & $82.8 \pm 15.5$ & $96.9 \pm 10.0$ \\
\hline \multicolumn{7}{|l|}{ Seminiferous tubules } \\
\hline Diameter $(\mu \mathrm{m})$ & $111 \pm 10^{\mathrm{a}}$ & $220 \pm 3$ & $214 \pm 4^{\mathrm{e}}$ & $137 \pm 10^{\mathrm{a}}$ & $226 \pm 9$ & $239 \pm 7$ \\
\hline Length (m per testis) & $24.8 \pm 2.7^{a}$ & $61.8 \pm 6.2$ & $62.3 \pm 3.3$ & $33.7 \pm 6.8^{\mathrm{a}}$ & $52.9 \pm 3.8$ & $68.2 \pm 7.2$ \\
\hline
\end{tabular}

Treated: the organ subjected to cryptorchidism or cryptorchidism followed by orchiopexy.

Contra: the scrotal organ contralateral to the treated side.

Control: normal age-matched control animals that did not undergo surgery.

A: type A spermatogonia; B: type B spermatogonia; PI-Z: preleptotene, leptotene and zygotene primary spermatocytes; P: pachytene primary spermatocytes; R-E: early spermatids (round and elongating spermatids); L: late elongated spermatids; o: cells rarely observed.

asignificantly different from contra values in the same treatment (cryptorchidism or orchiopexy) group (paired $t$ test, $P<0.05$ ).

bSignificantly different from control values in the same treatment group ( $t$ test, $P<0.05$ ).

cdesignificantly different from the corresponding treated/contra/control values in the orchiopexy group, respectively $(t$ test, $P<0.05)$.

using one-way ANOVA), the same statistical inferences would be reached except that the significant difference in the length of the seminiferous tubules between 'treated' and 'contra' animals in the orchiopexy group (Table 1) would not be detected (the significant difference between 'treated' and 'control' animals would be detected instead if this comparison was meaningful).

\section{Results}

\section{Comparison of control animals}

The age-matched control animals in the orchiopexy group were about 7 weeks older than those in the cryptorchidism group. There was a significant increase in testicular size $(30 \%)$ and diameter of the seminiferous tubules $(12 \%)$ (Table 1) with age. Such a trend was also apparent in the number of germ cells at advanced stages (Table 1): the total number of spermatids and spermatocytes per testis in the older group of animals $\left(2461 \pm 171 \times 10^{6}\right)$ was $45 \%$ greater $(P<0.05)$ than that in the younger group of animals $\left(1694 \pm 89 \times 10^{6}\right)$. The number of secondary spermatocytes (not shown in Table 1) as a percentage of all spermatocytes was $2.4 \pm 0.4 \%$ in the younger group and $2.3 \pm 0.8 \%$ in the older group. The number of Sertoli cells and the length of the seminiferous tubules per testis were did not change with age (Table 1).

The ratio of early spermatids: pachytene primary spermatocytes in the individual testes was significantly smaller in the younger group $(2.70 \pm 0.17)$ than in the older group $(3.45 \pm 0.26)(P<0.05)$.

Spermatozoa were densely packed in the epididymal tubules of control animals in both groups (Fig. 2a,b). Many round spermatids together with a few spermatocytes (mainly pachytene forms) were also observed in the younger group (Fig. 3a), whereas they were observed only occasionally in the older group (Fig. 3b). A preliminary study (not reported) showed that, in the younger group, a considerable number of round spermatids was observed in about $30 \%$ of the epididymal tubule sections, and the ratio of round spermatids:spermatozoa (estimated using the optical disector method) was $6.8 \%$ compared with only $0.13 \%$ in the older group.

\section{Comparison between the scrotal testis of unilaterally cryptorchid animals and the testis of age-matched control animals in the cryptorchidism group}

The comparison between the scrotal testis of unilaterally cryptorchid animals and the testis of age-matched control 

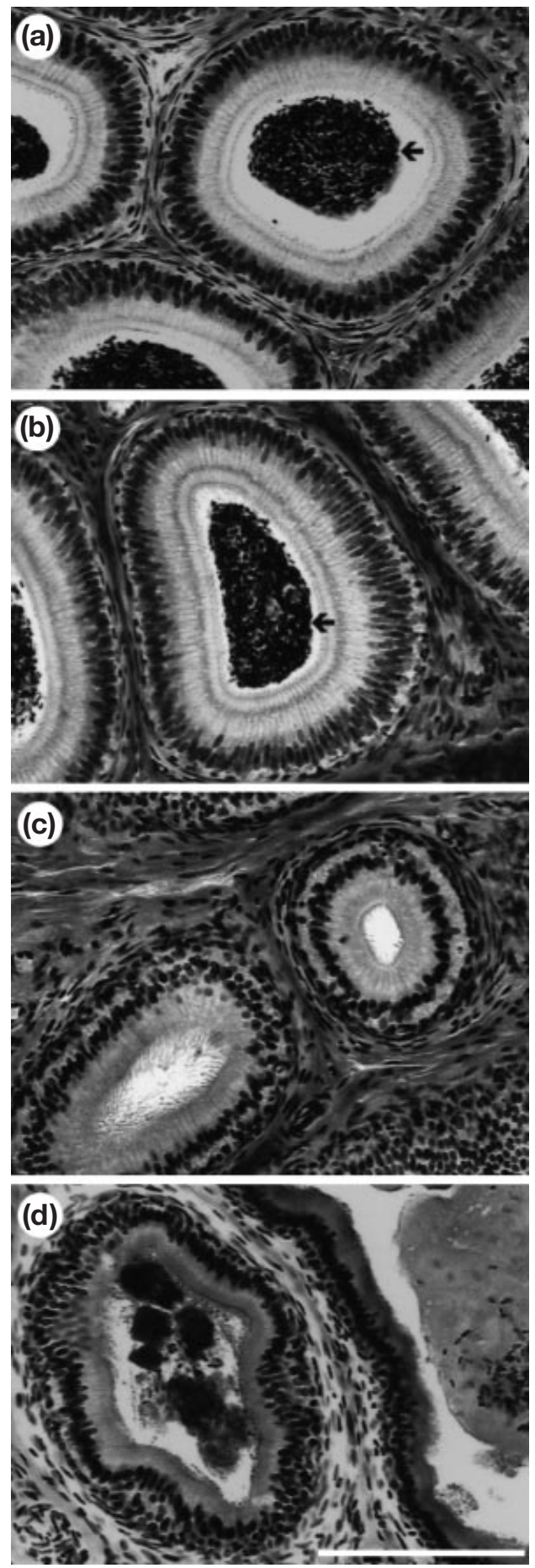

Fig. 2. Typical epididymal micrographs taken from (a) a control rabbit in the orchiopexy group that did not undergo surgery, (b) a control rabbit in the cryptorchidism group that did not undergo surgery, (c) the abdominal epididymis of a rabbit that underwent unilateral cryptorchidism and (d) the epididymis subjected to surgery from a rabbit that underwent unilateral cryptorchidism and subsequent orchiopexy. Note in the different sections the agglomerated spermatozoa (arrows; a,b), the absence of spermatozoa (c) and the interspersed spermatozoa (d) in the epididymal tubules. Scale bar represents $150 \mu \mathrm{m}$.
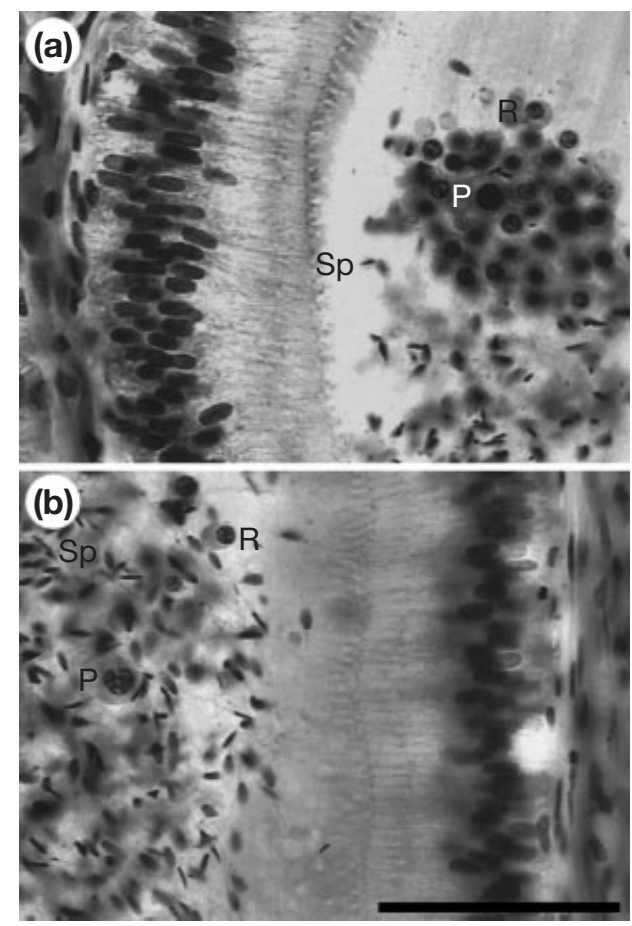

Fig. 3. Typical epididymal micrographs taken from control rabbits (not subjected to surgery) in (a) the cryptorchidism group and (b) the orchiopexy group. P: pachytene primary spermatocytes; R: round spermatids; Sp: spermatozoa. Scale bar represents $75 \mu \mathrm{m}$.

animals in the cryptorchidism group was undertaken to evaluate the effects of the unilaterally cryptorchid testis on the contralateral scrotal testis. The scrotal testis and epididymis of the animals with unilateral cryptorchidism were not significantly different from those of the control animals in all parameters investigated (Table 1) except in the number of late elongated spermatids, which was significantly smaller in the control animals.

Similar to findings from the epididymides of control animals in the cryptorchidism group (described above), a considerable number of round spermatids were observed in about $25 \%$ of the epididymal tubule sections in the scrotal epididymis of animals that underwent surgery (cryptorchidism group), and the ratio of round spermatids:spermatozoa was $9.6 \%$.

\section{Comparison between the scrotal testis of animals} undergoing orchiopexy and the testis of age-matched control animals in the orchiopexy group

The comparison between the scrotal testis of animals undergoing orchiopexy and the testis of age-matched control animals in the orchiopexy group was undertaken to evaluate the effects of the testis subjected to cryptorchidism and subsequent orchiopexy on the contralateral scrotal testis. Compared with the testis of control animals that did not undergo surgery, the scrotal testis of animals that 
underwent two operations (cryptorchidism and orchiopexy) was significantly smaller and had a significantly smaller total volume of seminiferous tubules (Table 1). The total number of all spermatocytes (including secondary spermatocytes) and early spermatids (not including late elongated spermatids) in the scrotal testis of animals that underwent surgery $\left(1368 \pm 170 \times 10^{6}\right.$ per testis $)$ was significantly $(P<0.05)$ decreased compared with that of controls $\left(1972 \pm 142 \times 10^{6}\right.$ per testis). The number of type B spermatogonia was also decreased, whereas the number of type A spermatogonia and Sertoli cells was unchanged (Table 1).

The epididymal volume and the total volume of epididymal tubules per scrotal epididymis in animals that underwent surgery appeared smaller than those in control animals, but this result did not reach significance (Table 1). Similar to findings in control animals in the orchiopexy group (described above), round spermatids were seen only occasionally and large numbers of spermatozoa were stored densely in the scrotal epididymis of animals that underwent surgery.

\section{Effects of cryptorchidism (cryptorchidism group)}

In animals in which cryptorchidism was induced, the abdominal testis was severely and consistently atrophied. The volume of the abdominal testis and the diameter and length (per testis) of the seminiferous tubules were only $22 \pm 3 \%$ (range $16-34 \%$ ), $50 \pm 5 \%$ (range $35-68 \%$ ) and $42 \pm 5 \%$ (range $17-56 \%$ ) of those of the scrotal testis, respectively (Table 1 ).

Numerous type B spermatogonia, spermatocytes and spermatids were observed in the scrotal testis, whereas these types of cell were essentially depleted in the abdominal testis; only a few atypical type B spermatogonia and spermatocytes were observed occasionally in the abdominal testis (Fig. 1c). The total number of spermatogonia and the number of type $A$ spermatogonia per abdominal testis were markedly and consistently reduced by $95 \pm 1 \%$ (range 92-97\%) and $84 \pm 3 \%$ (range 76-94\%), respectively (Table 1). However, the total number of Sertoli cells was unchanged (Table 1).

The abdominal epididymis was also atrophied: the volume of the abdominal epididymis and the total volume of the epididymal tubules were reduced significantly by $42 \%$ and $56 \%$, respectively (Table 1 ). Numerous spermatozoa were observed in the scrotal epididymis, as described above; however, spermatozoa were essentially absent from the abdominal epididymis (Fig. 2c). A few spermatozoa were observed on one of the three sections per epididymis after careful and thorough examination.

With respect to the immunocytochemistry, immunoreactivity was not apparent on control sections for which the primary antibody was omitted. Sertoli cell nuclei, which can be differentiated by shape, were not PCNA-positive. Small numbers of PCNA-positive germ cells (spermatogonia) were present in the abdominal testis. The profile number (per tubule cross-section) of PCNA-positive nuclei distributed along the tubule boundary was approximately $0.5 \pm 0.1$ in the abdominal testis, a value consistently and markedly smaller than that (approximately $13.3 \pm 0.5$; possibly including some early spermatocytes) in the scrotal testis.

\section{Effects of orchiopexy}

During orchiopexy, the cryptorchid testes were all markedly atrophied. The morphology of the cryptorchid testis and epididymis removed from one animal at this stage confirmed a histological change comparable to that seen in the cryptorchid testis in animals in the cryptorchidism group.

Compared with the contralateral scrotal testis, the testis and epididymis subjected to surgery remained atrophied after orchiopexy, and the total volume and length of the seminiferous tubules per testis and the total volume of the epididymal tubules remained markedly decreased (Table 1). Spermatogenesis was recovered only partially (compared with that in the cryptorchid testis in the cryptorchidism group) after orchiopexy and there was considerable variation in recovery among individuals.

In the non-treated scrotal testis of animals subjected to unilateral orchiopexy, the number of type A spermatogonia was $32 \pm 3 \times 10^{6}$ (range 22-42) per testis, the total number of spermatocytes was $437 \pm 40 \times 10^{6}$ (range 320-569) per testis, the number of early spermatids was $931 \pm 141 \times 10^{6}$ (range 506-1382) per testis, and the diameter of the seminiferous tubules was $226 \pm 9 \mu \mathrm{m}$ (range 198-253). In the contralateral treated testis of five animals that underwent orchiopexy, the number of Sertoli cells per testis remained unchanged (Table 1). However, marked and variable changes occurred in other morphological and morphometric characteristics. (i) In one animal, only a few spermatogonia and early spermatocytes were observed, no spermatids were present, and the total number of spermatogonia and spermatocytes was $<6 \times 10^{6}$ per testis, similar to findings in the abdominal testis in animals in the cryptorchidism group. Spermatozoa and round spermatids were essentially absent from the epididymis. (ii) In another animal, the number of type $A$ spermatogonia returned to $18 \times 10^{6}$ per testis, but the total number of type $B$ spermatogonia and all spermatocytes (spermatids absent) was $<15 \times 10^{6}$ per testis (Fig. 1d). Spermatozoa and round spermatids were also essentially absent from the epididymis. (iii) In another two animals, although spermatids were absent, many spermatocytes (156-157 × 106 per testis) could be seen and the number of type $A$ spermatogonia per testis returned to $20-34 \times 10^{6}$ per testis (Fig. 1e). In the epididymis of one animal, spermatozoa and round spermatids were essentially absent, whereas in the epididymis of the other animal some round spermatids were observed and spermatozoa were occasionally present. (iv) In the fifth animal, all types of germ cell were present but the late elongated spermatids were observed only occasionally (Fig. 1f). The numbers of type A spermatogonia, all sperma- 
tocytes and all spermatids were 21, 169 and $108 \times 10^{6}$ per testis, respectively. The diameter of the seminiferous tubules was $166 \mu \mathrm{m}$ (those of the above four animals were between 107 and $146 \mu \mathrm{m})$. Round spermatids and spermatozoa were observed in the epididymis (Fig. 2d).

On average, the profile number (per tubule cross-section) of PCNA-positive nuclei distributed along the tubule boundary was approximately $7.0 \pm 1.2$ in the testis of animals subjected to orchiopexy, which was significantly lower than that $(18.1 \pm 1.3)$ in the contralateral scrotal testis.

\section{Discussion}

This is the first study to report the absolute numbers of different types of germ cell and Sertoli cells, obtained using an unbiased and efficient stereological tool (the optical disector), in the rabbit testis with or without cryptorchidism and orchiopexy. The study demonstrates that induction of abdominal cryptorchidism in mature rabbits for 13 weeks leads to testicular atrophy and spermatogenic arrest at type A spermatogonia; the number of type A spermatogonia per testis in cryptorchid animals was markedly and consistently reduced by $84 \%$. With subsequent orchiopexy for 7 weeks, the testis remained atrophied and the number of type $A$ spermatogonia returned to the near-normal range in four of five animals, but spermatogenesis recovered only partially at the stage of early primary spermatocytes (one animal), late primary spermatocytes (two animals) or spermatids (one animal). It is speculated that cryptorchidism resulted in spermatogenic arrest that was potentially recoverable (in view of the restoration of the number of type A spermatogonia), but that orchiopexy failed to provide an ideal environment for full recovery of spermatogenesis, probably because the surgery had considerable iatrogenic effects on the blood supply or innervation to the testis and did not enable reconstruction of a serous sac around the testis without adhesions, which may be important for normal spermatogenesis.

In support of this study, orchiopexy after cryptorchidism in adult rats also failed to result in full recovery of spermatogenesis (cryptorchidism for 10 and 28 days, and orchiopexy for 28 days; Jégou et al., 1983) or reversible azoospermia (animals were vasocystostomized for 4 weeks to enable sperm output measurements before cryptorchidism was induced for 2-8 weeks, followed by orchiopexy for 3 months; Kort et al., 1990). Nishimune et al. (1978) reported full recovery of spermatogenesis after orchiopexy (for 2 months) in the cryptorchid (2 months) adult mice model; however, their conclusions were based only on histological observation and no supporting quantitative data were provided.

The present study also showed that inducing unilateral cryptorchidism in one testis for 13 weeks had no effect on spermatogenesis in the contralateral scrotal testis, except that significantly more late elongated spermatids were found in the scrotal testis of animals that underwent surgery compared with control animals. However, after a second surgical procedure (orchiopexy for 7 weeks), a significant decrease in the numbers of all spermatocytes and early spermatids was observed. Despite the decrease, the number of the late elongated spermatids in the scrotal testis of animals that underwent surgery was not decreased compared with that in control animals. The ratio of late elongated spermatids: early spermatids was $47 \pm 6 \%$ for the scrotal testis of animals that underwent surgery and $36 \pm 4 \%$ for the control animals in the cryptorchidism group. Similarly, in the orchiopexy group, the ratio $(45 \pm 5 \%)$ of these cells in the scrotal testis of animals that underwent surgery also appeared to be larger than that $(35 \pm 3 \%)$ in control animals, although this result failed to reach significance in either group. Therefore, it is concluded that performing surgery on one testis may reduce the production of spermatocytes and early spermatids in the contralateral testis (not subjected to surgery) and that the reduction is associated with a delayed release of late elongated spermatids, a situation that is observed with gonadotrophin withdrawal in humans (Zhengwei et al., 1998b) and monkeys (Zhengwei et al., 1998a). Zhengwei et al. (1998a) reported a significant decrease in serum inhibin concentrations 16 days after placebo injection in adult male monkeys. Pryor et al. (1989) reported that sham operation (disection and slight manipulation of the testis) significantly reduced the diameter of the seminiferous tubules by $32 \%$ and the tubular fertility scores by $42 \%$, compared with those in control adult rabbits not subjected to surgery. Taken together, these results indicate that it may be the severe surgical trauma or associated emotional changes (for example anxiety), not the unilateral cryptorchidism and orchiopexy, which, at least in part, induced the changes in spermatogenesis described above.

On the basis of the lower ratio of spermatids (daughter cells): primary spermatocytes (progenitor cells) (the maximum theoretical value of the ratio is 4), other studies, including that by Sharpe (1994), have concluded that degeneration of spermatids during meiosis is common in the normal testes of most mammals. For example, the calculated shortfall was as high as $39 \%$ in men, $20 \%$ in bulls and $24 \%$ in rabbits. However, this conclusion can be challenged in three respects in terms of: (i) whether the numbers of cells were obtained using an unbiased method; (ii) whether the duration of the daughter cells and the progenitor cells in the spermatogenic cycle was taken into consideration (Zhengwei et al., 1997); and (iii) whether immature spermatids were sloughing off the seminiferous epithelium and leaving the testis before maturation.

In the present study, the ratio of early spermatids: pachytene spermatocytes was estimated to be $2.70 \pm 0.17$ for control animals in the cryptorchidism group and $3.45 \pm 0.26$ for control animals in the orchiopexy group. As a greater number of round spermatids in the epididymis was observed in the cryptorchidism group, the significantly lower ratio for this group (relative to 
the theoretical value of 4 and to the ratio for the orchiopexy group) may be the result of sloughing of spermatids. In studies in which the same stereological methods were used and the same types of cell were counted, the ratio was $3.94 \pm 0.19(n=6)$ in normal monkeys (Zhengwei et al., $1997)$ and $3.71 \pm 0.41(n=5)$ in normal men (Zhengwei et al., 1998b; Yang and Wen, 1999). Round spermatids were rarely seen in the epididymides of the monkeys (Wen and Yang, 2000); round spermatids (as identified with immunocytochemical staining) were also observed only occasionally in semen from normal men (Zhengwei et al., 1998b,c). In another study (in which epididymal histology and semen profile were not investigated), the ratio of early spermatids:pachytene spermatocytes, estimated using the same methods, was found to be $4.08 \pm 0.27(n=4)$ and $4.08 \pm 0.33(n=5)$ for the two groups of control monkeys (Zhengwei et al., 1998a).

With respect to the different degrees of sloughing of spermatids in the two groups of control animals, in the cryptorchidism group (in which sloughing of spermatids was more apparent), organs were removed from control animals in September (late summer) when the animals were about 7-8 months old, whereas, in the orchiopexy group, organs were removed in May (spring) and the animals were 7 weeks older. There was a significant increase in both testicular size (by 30\%) and the total number of spermatids and all spermatocytes per testis (by 45\%) in the older group of animals, which indicates that, in the younger animals, spermatogenesis was actively occuring. Furthermore, in two animals that underwent orchiopexy, groups of round spermatids were observed in the epididymis subjected to surgery, and spermatogenesis in the ipsilateral testes of these two animals was partially recovered after spermatogenic arrest induced by cryptorchidism. Thus it is possible that age or season contributes to sloughing of spermatids, which might occur more frequently after puberty or when spermatogenesis starts to occur actively.

The authors are grateful to the reviewers for suggesting that orchiopexy should be included in the study. This study was supported by an Excellent Young Researcher Grant from the Chinese Ministry of Education and grants from the Sichuan Youth Foundation of Science and Technology and the Sichuan Committee of Family Planning.

\section{References}

Bar-Maor JA, Nissan S, Lernau OZ, Oren M and Levy E (1979) Orchidopexy in cryptorchidism assessed by clinical, histologic and sperm examinations Surgery, Gynecology and Obstetrics 148 855-859

Boekelheide K, Eveleth J and Hall SJ (1990) Experimental cryptorchidism protects against long-term 2,5-hexanedione-induced testicular germ cell loss in the rat Journal of Andrology 11 105-112

Cortes D, Thorup JM and Lindenberg S (1996) Fertility potential after unilateral orchiopexy: an age-independent risk of subsequent infertility when biopsies at surgery lack germ cells Journal of Urology 156 $217-220$

Foresta C, Ferlin A, Garolla A, Milani C, Oliva G and Rossato M (1996) Functional and cytologic features of the contralateral testis in cryptorchidism Fertility and Sterility $\mathbf{6 6} 624-629$
Gracia J, Sanchez J, Garcia C, Pueyo C and Ferrandez A (1998) What is the relationship between spermatozoa per milliliter at adulthood and the tubular fertility index at surgical age for patients with cryptorchidism? Journal of Pediatric Surgery 33 594-596

Gundersen HJG (1977) Notes on the estimation of the numerical density of arbitrary profiles: the edge effect Journal of Microscopy 111 219-223

Hodgman CD, Weast RC and Selby SM (1958) Handbook of Chemistry and Physics pp 2083-2089 Chemical Rubber Publishing Co., Cleveland, $\mathrm{OH}$

Huff DS, Fenig DM, Canning DA, Carr MG, Zderic SA and Snyder HM III (2001) Abnormal germ cell development in cryptorchidism Hormone Research 55 11-17

Jégou B, Laws AO and de Kretser DM (1983) The effect of cryptorchidism and subsequent orchidopexy on testicular function in adult rats Journal of Reproduction and Fertility 69 137-145

Kort WJ, Hekking-Weijma I and Vermeij M (1990) Artificial intraabdominal cryptorchidism in young adult rats leads to irreversible azoospermia European Urology 18 302-306

Lunstra DD and Schanbacher BD (1988) Testicular function and Leydig cell ultrastructure in long-term bilaterally cryptorchid rams Biology of Reproduction 38 211-220

Mieusset R, Grandjean H, Mansat A and Pontonnier F (1985) Inhibiting effect of artificial cryptorchidism on spermatogenesis Fertility and Sterility 43 589-594

Nishimune Y, Aizawa S and Komatsu T (1978) Testicular germ cell differentiation in vivo. Fertility and Sterility 29 95-102

Penson DF, Lugg JA, Coyne C, Sadeghi F, Freedman AL, Gonzalez-Cadavid NF and Rajfer J (1997) Effect of cryptorchidism on testicular histology in a naturally cryptorchid animal model Journal of Urology 158 1978-1982

Pryor JL, Hurt GS, Caloras D, Turner TT, Flickinger CJ and Howards SS (1989) Histologic analysis of orchiopexy in a cryptorchid rabbit model Journal of Urology 142 413-417

Quinn FM (1991) Evaluation of the scrotal testis before and after orchidopexy in experimental unilateral cryptorchidism Journal of Pediatric Surgery 26 602-606

Rogers E, Teahan S, Gallagher H, Butler MR, Grainger R, McDermott TE and Thornhill JA (1998) The role of orchiectomy in the management of postpubertal cryptorchidism Journal of Urology 159 851-854

Rune GM, Mayr J, Neugebauer H, Anders C and Sauer H (1992) Pattern of Sertoli cell degeneration in cryptorchid prepubertal testes International Journal of Andrology 15 19-31

Saito $\mathbf{S}$ and Kumamoto $\mathbf{Y}(1989)$ The number of spermatogonia in various congenital testicular disorders Journal of Urology 141 1166-1168

Salman FT, Adkins ES and Fonkalsrud EW (1988) Morphologic effects of unilateral cryptorchidism on the contralateral descended testis Journal of Pediatric Surgery 23 439-443

Sharpe RM (1994) Regulation of spermatogenesis. In The Physiology of Reproduction pp 1363-1434 Eds E Knobil and JD Neill. Raven Press, New York

Sterio DC (1984) The unbiased estimation of number and sizes of arbitrary particles using the disector Journal of Microscopy 134 127-136

Swierstra EE and Foote RH (1963) Cytology and kinetics of spermatogenesis in the rabbit Journal of Reproduction and Fertility 5 309-322

Wen XH and Yang ZW (2000) Quantitative (stereological) study on the spermatozoal storage capacity of epididymis in rats and monkeys Asian Journal of Andrology 2 73-77

Wen XH, Wang XH, Tong JS, Yang ZW and Zhang GY (2000) Reversible effect of testosterone undecanoate injection on spermatogenesis in rats Asian Journal of Andrology 2 207-211

Wreford NG (1995) Theory and practice of stereological techniques applied to the estimation of cell number and nuclear volume in the testis Microscopy Research and Technique 32 423-436

Yang ZW and Wen XH (1999) A quantitative study on the efficiency of meiosis during spermatogenesis in normal adult men Chinese Journal of Stereology and Image Analysis 4 1-5

Yin Y, Hawkins KL, DeWolf WC and Morgentaler A (1997) Heat stress causes testicular germ cell apoptosis in adult mice Journal of Andrology 18 159-165 
Zhengwei Y, McLachlan RI, Bremner WJ and Wreford NG (1997) Quantitative (stereological) study of normal spermatogenesis in the adult monkey (Macaca fascicularis) Journal of Andrology 18 681-687

Zhengwei Y, Wreford NG, Schlatt S, Weinbauer GF, Nieschlag E and McLachlan RI (1998a) Acute and specific impairment of spermatogonial development by GnRH antagonist-induced gonadotrophin withdrawal in the adult macaque (Macaca fascicularis) Journal of Reproduction and Fertility 112 139-147

Zhengwei Y, Wreford NG, Royce P, de Kretser DM and McLachlan RI (1998b) Stereological evaluation of human spermatogenesis after suppression by testosterone treatment: heterogeneous pattern of spermato- genic impairment Journal of Clinical Endocrinology and Metabolism 83 1284-1291

Zhengwei Y, Wreford NG, Bremner WJ, Matsumoto AM, Anawalt BA and McLachlan RI (1998c) Immature spermatids are not prevalent in semen from men who are receiving androgen-based contraceptive regimens Fertility and Sterility 69 89-95

Received 5 December 2001.

First decision 8 February 2002.

Accepted 3 March 2002. 